\title{
The influence of organizational context on the use of research by nurses in Canadian pediatric hospitals
}

\author{
Janet E Squires ${ }^{1,2^{*}}$, Carole A Estabrooks ${ }^{3}$, Shannon D Scott ${ }^{3}$, Greta G Cummings ${ }^{3}$, Leslie Hayduk4, \\ Sung Hyun Kang ${ }^{3}$ and Bonnie Stevens ${ }^{5,6}$
}

\begin{abstract}
Background: Organizational context is recognized as an important influence on the successful implementation of research by healthcare professionals. However, there is relatively little empirical evidence to support this widely held view.

Methods: The objective of this study was to identify dimensions of organizational context and individual (nurse) characteristics that influence pediatric nurses' self-reported use of research. Data on research use, individual, and contextual variables were collected from registered nurses $(N=735)$ working on 32 medical, surgical and critical care units in eight Canadian pediatric hospitals using an online survey. We used Generalized Estimating Equation modeling to account for the correlated structure of the data and to identify which contextual dimensions and individual characteristics predict two kinds of self-reported research use: instrumental (direct) and conceptual (indirect).

Results: Significant predictors of instrumental research use included: at the individual level; belief suspension-implement, research use in the past, and at the hospital unit (context) level; culture, and the proportion on nurses possessing a baccalaureate degree or higher. Significant predictors of conceptual research use included: at the individual nurse level; belief suspension-implement, problem solving ability, use of research in the past, and at the hospital unit (context) level; leadership, culture, evaluation, formal interactions, informal interactions, organizational slack-space, and unit specialty.

Conclusions: Hospitals, by focusing attention on modifiable elements of unit context may positively influence nurses' reported use of research. This influence of context may extend to the adoption of best practices in general and other innovative or quality interventions.
\end{abstract}

\section{Background}

Pediatrics is not immune to the well-documented challenges of successful implementation of quality improvement initiatives, moving research evidence into practice and other knowledge translation efforts. Many reasons have been cited for the gap between the research evidence and what occurs in clinical practice including lack of research studies in child health to inform practice [1], healthcare professionals' knowledge of the evidence, and how contextual factors impend or facilitate the implementation of research [2]. Yet, in the conventional

\footnotetext{
* Correspondence: jasquires@ohri.ca

'Clinical Epidemiology Program, Ottawa Hospital Research Institute, Ottawa, Canada

${ }^{2}$ School of Nursing, Faculty of Health Sciences, University of Ottawa, Ottawa, Canada

Full list of author information is available at the end of the article
}

pediatric health literature, the overwhelming focus in the implementation of research is on patient and provider outcomes with little mention of how the practice environment (or context) shapes the process of implementation [3]. One notable exception is in the pediatric social service literature where there is evidence that organizational 'social' context (defined as climate and culture) of the service system shapes the nature of the services provided by the professionals working in those systems [3-5]. Yet, there are many examples in the pediatric health literature of research-based implementations focused on specific clinical conditions such as asthma and pain management, occurring in multiple health care environments, with no assessment of contextual factors. In these situations typically provider and patient outcomes [6-9] are the focus. This research approach implicitly suggests that context is a constant or exerts little influence on implementation

\section{Biomed Central}


success; however, a rapidly emerging body of literature suggests otherwise and stresses the powerful influence of context $[10,11]$.

Organizational context is now recognized as an important influence on the successful implementation of, or failure to adopt, quality improvement initiatives, research evidence, and best practices [12-14]. Recent syntheses have led to the development of theory describing the relationships among contextual factors that lead to successful implementation of innovation and quality improvement generally [15-18]. Less understood are: (1) which contextual factors influence different kinds of research use; (2) what level of context (e.g., hospital facility or unit) is most relevant to successful adoption of research; and, (3) the interacting influences that may be at play between different organizational (e.g., unit vs. hospital) levels. Microsystems theory adds a useful perspective [19-24]. This theory describes organizations as comprised of macrosystems comprised of mesosytems (i.e., centres and programs), which, in turn, consist of interrelated microsystems or clinical units. Clinical units are the 'ground zero' of patient care delivery, the essential building blocks of organizations [19-24]. As a result, strategies focused on the clinical unit have potential to enhance the adoption of research and ultimately to transform healthcare systems [22]. The microsystem literature acknowledges the influence of unit context [19] on outcomes and patient experiences with growing evidence that the unit is the interface at which quality patient outcomes are best achieved [22,25].

In this paper, we address the gap in knowledge related to the influence of contextual factors on nurses' use of research evidence in pediatric settings. Notably, we build on our prior pilot study [26] where we found that a more positive perception of a unit's leadership, culture, and evaluation was associated with higher self-reported use of research findings in practice [26]. Research use is a multidimensional construct that consists of three kinds of research use: instrumental, conceptual, and symbolic (or persuasive) [27-29]. Instrumental research use is a direct use of research knowledge. It refers to the concrete application of research in clinical practice, either in making specific decisions or as knowledge to guide specific interventions related to patient care. Conceptual research use is an indirect application of research; it refers to the cognitive use of research where the research findings may change one's opinion or mind set about a specific practice area but not necessarily one's particular action. Finally, symbolic (or persuasive) research utilization is the use of research knowledge as a political tool in order to influence policies and decisions or to legitimate a position [27-29]. The purpose of the study reported here is to identify dimensions of organizational context and individual (nurse) characteristics that influence pediatric nurses' self-reported use of research. Specifically, we focus on instrumental and conceptual use of research.

\section{Methods}

\section{Design and sample, and data collection}

The study reported in this paper was conducted as part of the Translating Research on Pain in Children (TROPIC) project within the Canadian Institutes for Health Research (CIHR) Team in Children's Pain research program. We used a cross-sectional (survey) design. Of the 15 children hospitals in Canada at the start of the study, eight met our requirements of having four or more distinct units, excluding psychiatric and emergency units, with 30 or more beds. Psychiatric and emergency units were ineligible because of the potential for adverse psychologic responses to pain and/or the low incidence of painful procedures on these units [30]. The eight eligible sites were all urban-based universityaffiliated pediatric hospitals; all eight sites agreed to participate. Study units within each site were eligible for inclusion if they had a distinct geographic location and administrative structure; admitted children for periods longer than 24 hours; administered painful procedures to inpatients; and had pharmacologic, physical and psychologic interventions available for managing pain [30]. For sites with more than four eligible units, four units were randomly selected to participate to include at least one medical, one surgical and one critical care unit [30].

Data were collected for the current study at two levels: (1) clinical unit, and (2) individual healthcare professionals. Some unit (e.g., average length of patient stay) data were collected using the TROPIC unit profile survey, which was completed electronically by a research nurse at each participating hospital. Individual data, some later aggregated to the unit level, came from staff (survey) and patients (chart abstraction). Healthcare professionals (nurses, allied, practice specialists, physicians) and unit managers that met the study inclusion criteria (Additional file 1) and who could be contacted were invited to complete the TROPIC staff survey on two occasions: Time 1 (May - August 2008) and Time 2 (April - August 2011). The same units were sampled on both time points but individuals completing the survey were not linked; preventing us from being able to combine data or compare respondents across time. The survey was completed online with participant responses compiled in a centralized database. A research nurse was present on the included units on a selection of all shifts (day, evening, night) for the full data collection period (3 months) to answer questions about the study and provide eligible participants with a survey package containing a letter introducing the study, and a business card providing a Uniform Resource Locator (URL) and 
unique password to access the survey on-line. In this paper we report analyses that used the most current data -- staff survey (administered to nurses) and unit profile survey collected at Time 2; return of the surveys implied consent to participate.

\section{Ethics}

Ethical approvals for this study were obtained from the Health Research Ethics Boards of the appropriate Canadian universities (application Pro00003308), as well as, the hospital ethics review boards (where applicable) for all hospitals participating in the study. All research was in compliance with the Helsinki Declaration (http://www. wma.net/en/30publications/10policies/b3/index.html).

\section{Study variables}

\section{Dependent variables}

The dependent variables assessed in this study were instrumental research use (IRU) and conceptual research use (CRU). They are both included in the staff survey. IRU refers to a direct and concrete use of research evidence in practice (e.g., use of guidelines and protocols) [31]. CRU refers to the cognitive use of research where the research findings may change one's opinion or mind set about a specific practice area but not necessarily one's particular action [28,31]. Both variables were measured using single items scored on five-point frequency scales from ' $10 \%$ or less of the time' to 'almost $100 \%$ of the time'. A recent systematic review [32] indicates that these measures have been used previously to obtain reliable and valid assessments of research utilization from nurses.

\section{Explanatory variables}

Our explanatory variables are listed in Tables 1 and 2 . We selected these variables based on those available to us in the unit profile and staff surveys. Each variable was assigned to individual- or unit-level measurement (Table 3) in a series of team meetings. One variable, unit specialty (medicine, surgery, or critical care), was assigned as part of our sampling frame to ensure equal representation across specialties. Details on all explanatory variables, including definitions, measurement, and reliability, is presented in Additional file 2.

From the unit profile survey we obtained the following two variables (each assigned as unit-level measurement in our models): (1) average number of beds occupied and (2) average length of patient stay (in days). The remaining variables were obtained from the staff survey. Unit-level variables included: context $(n=10$ variables, as measured by the Alberta Context Tool), support for innovation, and percentage of nurses on the unit with a baccalaureate or higher degree.

Context Context was measured using the Alberta Context Tool (ACT) [33], premised on the Promoting

Table 1 Characteristics of nurse respondents for outcome and categorical covariates $(\mathbf{N}=735)$

\begin{tabular}{|c|c|c|c|c|}
\hline \multicolumn{2}{|c|}{ Variables } & $\mathrm{N}(\%)$ & $\begin{array}{c}\text { IRU } \\
\text { Mean (SD) }\end{array}$ & $\begin{array}{c}\text { CRU } \\
\text { Mean (SD) }\end{array}$ \\
\hline \multirow[t]{5}{*}{ Age } & $20-29$ years & $250(34.1)$ & $3.831(1.04)$ & $3.620(1.15)$ \\
\hline & 30-39 years & $198(27.0)$ & $3.657(1.11)$ & 3.465 (1.18) \\
\hline & 40-49 years & $151(20.6)$ & $3.313(1.22)$ & $3.424(1.20)$ \\
\hline & $50-59$ years & $120(16.3)$ & $3.425(1.31)$ & $3.500(1.26)$ \\
\hline & $\geq 60$ years & $15(2.0)$ & $2.800(1.32)$ & $3.467(1.13)$ \\
\hline \multirow[t]{2}{*}{ Sex } & Male & $42(5.7)$ & $3.452(1.04)$ & $3.119(1.37)$ \\
\hline & Female & $692(94.1)$ & $3.600(1.18)$ & $3.542(1.17)$ \\
\hline \multirow[t]{3}{*}{ Education } & Diploma/Certificate & $212(28.9)$ & $3.175(1.27)$ & $3.292(1.25)$ \\
\hline & Bachelor & $496(67.7)$ & $3.769(1.07)$ & $3.619(1.15)$ \\
\hline & Master or higher & $25(3.4)$ & $3.520(1.19)$ & $3.440(1.12)$ \\
\hline \multirow[t]{3}{*}{ Employment status } & Full-Time & $450(61.3)$ & $3.690(1.10)$ & $3.636(1.13)$ \\
\hline & Part-Time & $254(34.6)$ & $3.480(1.25)$ & $3.331(1.27)$ \\
\hline & Casual & $30(4.1)$ & $3.067(1.31)$ & $3.333(0.99)$ \\
\hline \multirow[t]{3}{*}{ Specialty } & Surgical & $141(19.2)$ & $3.604(1.13)$ & $3.482(1.23)$ \\
\hline & Medical & $276(37.6)$ & $3.598(1.19)$ & $3.489(1.21)$ \\
\hline & Critical Care & $318(43.3)$ & $3.579(1.17)$ & $3.553(1.14)$ \\
\hline \multirow[t]{2}{*}{ Specialized course } & Yes & $222(30.2)$ & $3.694(1.14)$ & $3.703(1.09)$ \\
\hline & No & $513(69.8)$ & $3.546(1.18)$ & $3.435(1.22)$ \\
\hline
\end{tabular}


Table 2 Descriptive results for continuous covariates $(N=748)$

\begin{tabular}{|c|c|c|c|c|c|c|}
\hline \multirow[t]{2}{*}{ Variables } & \multirow[t]{2}{*}{ Mean (SD) } & \multicolumn{5}{|c|}{ Aggregation Statistics } \\
\hline & & $\mathrm{ICC}(1)$ & $\operatorname{ICC}(2)$ & $\eta^{2}$ & $\omega^{2}$ & $p$ \\
\hline Attitude towards research & $4.188(0.47)$ & 0.027 & 0.387 & 0.068 & 0.026 & 0.017 \\
\hline Belief suspension (Willingness) & $3.956(0.66)$ & 0.041 & 0.494 & 0.081 & 0.040 & 0.001 \\
\hline Belief suspension (Implement) & $3.592(0.86)$ & 0.068 & 0.622 & 0.105 & 0.065 & 0.000 \\
\hline SF-8 ${ }^{\text {TM }}$ (Physical health status) & $50.107(7.53)$ & 0.018 & 0.296 & 0.060 & 0.018 & 0.066 \\
\hline SF-8 ${ }^{\mathrm{TM}}$ (Mental health status) & $48.397(8.94)$ & 0.016 & 0.268 & 0.057 & 0.015 & 0.091 \\
\hline MBI Exhaustion & $2.150(1.18)$ & 0.077 & 0.653 & 0.113 & 0.074 & 0.000 \\
\hline MBI Cynicism & $1.739(1.19)$ & 0.031 & 0.421 & 0.071 & 0.030 & 0.009 \\
\hline MBI Efficacy & $4.252(1.00)$ & 0.054 & 0.562 & 0.092 & 0.052 & 0.000 \\
\hline Adequate orientation & $4.018(0.75)$ & 0.023 & 0.349 & 0.063 & 0.022 & 0.033 \\
\hline Job satisfaction & $4.007(0.77)$ & 0.061 & 0.597 & 0.099 & 0.059 & 0.000 \\
\hline Problem solving & $3.825(0.36)$ & 0.000 & 0.000 & 0.039 & 0.000 & 0.613 \\
\hline Research use in past & $4.004(0.72)$ & 0.031 & 0.418 & 0.071 & 0.029 & 0.010 \\
\hline ACT Leadership & $3.719(0.76)$ & 0.066 & 0.614 & 0.104 & 0.064 & 0.000 \\
\hline ACT Culture & $3.848(0.54)$ & 0.101 & 0.718 & 0.137 & 0.098 & 0.000 \\
\hline ACT Evaluation & $3.181(0.83)$ & 0.318 & 0.913 & 0.340 & 0.310 & 0.000 \\
\hline ACT Formal Interactions & $1.798(1.01)$ & 0.144 & 0.793 & 0.176 & 0.140 & 0.000 \\
\hline ACT Informal Interactions & $5.376(1.66)$ & 0.145 & 0.794 & 0.179 & 0.142 & 0.000 \\
\hline ACT Social Capital & $3.973(0.48)$ & 0.052 & 0.556 & 0.091 & 0.051 & 0.000 \\
\hline ACT Structural and Electronic Resources & $4.921(1.78)$ & 0.078 & 0.658 & 0.116 & 0.076 & 0.000 \\
\hline ACT Organizational Slack-Staff & $3.138(0.98)$ & 0.317 & 0.913 & 0.338 & 0.308 & 0.000 \\
\hline ACT Organizational Slack-Space & $2.980(0.89)$ & 0.215 & 0.861 & 0.243 & 0.209 & 0.000 \\
\hline ACT Organizational Slack-Time & $2.999(0.60)$ & 0.205 & 0.854 & 0.232 & 0.198 & 0.000 \\
\hline Support for innovation & $3.456(0.83)$ & 0.086 & 0.682 & 0.122 & 0.083 & 0.000 \\
\hline$\%$ of baccalaureate & $0.711(0.45)$ & N/A & N/A & N/A & N/A & N/A \\
\hline \# of average occupied beds & $25.287(12.60)$ & N/A & N/A & N/A & N/A & N/A \\
\hline Average patient stay & $7.600(6.03)$ & N/A & $\mathrm{N} / \mathrm{A}$ & N/A & N/A & N/A \\
\hline
\end{tabular}

Action on Research Implementation in Health Services framework which argues that successful implementation of research is a function of optimal levels of context, facilitation and evidence [34,35]. The Pediatric Nurse version of the ACT contained in the staff survey contains 58 items, which reflect 10 organizational context concepts: leadership, culture, evaluation, social capital, informal interactions, formal interactions, resources, and organizational slack (staff, space, and time). Scores on the ACT are obtained from individuals (in this case, nurses) and aggregated to generate unit scores for each of the 10 dimensions of the measure. Reliability and validity of scores with nurses was demonstrated previously using Time 1 data from this study [33]. In that analysis, a principal components analysis indicated a 13-factor solution. Bivariate associations between IRU (which the ACT was developed to predict) and the majority of ACT factors were statistically significant supporting construct validity. Adequate internal consistency reliability was also reported [33]. Reliability coefficients using Time 2 data (used in this paper) is also available as part of the instrument table in Additional file 2.

Individual variables Demographic variables included were: age, sex, highest education, and employment status. Individual variables included were: attitude towards research, belief suspension-implement, belief suspensionwillingness, physical health status, mental health status, three dimensions of burnout (exhaustion, cynicism, and efficacy), adequate orientation, job satisfaction, problem solving, attendance at specialized courses, and research use in the past.

\section{Analytic strategy}

Reliability and validity of aggregated data at the unit level Contextual concepts were hypothesized as unit-level concepts (i.e., scores on these variables were believed to be similar among nurses in a unit). While direct 
Table 3 GEE Results for Instrumental Research Use (IRU) and Conceptual Research Use (CRU) (Independent Working Correlation Structure)

\begin{tabular}{|c|c|c|c|c|c|}
\hline \multirow[t]{2}{*}{ Level } & \multirow[t]{2}{*}{ Variables } & \multicolumn{2}{|c|}{ IRU } & \multicolumn{2}{|c|}{ CRU } \\
\hline & & Estimate (SE) & p-value & Estimate (SE) & p-value \\
\hline \multirow[t]{19}{*}{ Individual level covariates $(\mathrm{N}=748)$} & Attitude towards research & $0.168(0.107)$ & 0.118 & $0.035(0.095)$ & 0.712 \\
\hline & Belief suspension (Implement) & $0.159(0.054)$ & 0.004 & $0.142(0.062)$ & 0.023 \\
\hline & Belief suspension (Willingness) & $-0.020(0.050)$ & 0.687 & $-0.003(0.074)$ & 0.968 \\
\hline & SF-8 ${ }^{\mathrm{TM}}$ (Physical health status) & $0.005(0.006)$ & 0.414 & $-0.002(0.007)$ & 0.812 \\
\hline & SF-8 ${ }^{\mathrm{TM}}$ (Mental health status) & $-0.001(0.005)$ & 0.839 & $-0.001(0.005)$ & 0.921 \\
\hline & MBI Emotional Exhaustion & $0.063(0.057)$ & 0.266 & $0.072(0.054)$ & 0.184 \\
\hline & MBI Cynicism & $-0.118(0.050)$ & 0.017 & $-0.075(0.047)$ & 0.111 \\
\hline & MBI Efficacy & $0.065(0.036)$ & 0.071 & $0.058(0.043)$ & 0.174 \\
\hline & Adequate orientation & $0.117(0.066)$ & 0.074 & $0.047(0.063)$ & 0.455 \\
\hline & Job satisfaction & $0.069(0.067)$ & 0.305 & $0.152(0.078)$ & 0.050 \\
\hline & $\mathrm{Age}^{1}$ & $-0.023(0.025)$ & 0.347 & $0.018(0.023)$ & 0.442 \\
\hline & $\operatorname{Sex}^{2}$ & $-0.152(0.193)$ & 0.431 & $-0.232(0.225)$ & 0.302 \\
\hline & Highest education - Diploma/Certificate ${ }^{3}$ & $-0.032(0.219)$ & 0.882 & $0.074(0.235)$ & 0.752 \\
\hline & Bachelor degree & $0.376(0.223)$ & 0.091 & $0.287(0.233)$ & 0.219 \\
\hline & Employment status - Full time ${ }^{4}$ & $0.290(0.275)$ & 0.291 & $0.137(0.245)$ & 0.576 \\
\hline & Part time & $0.294(0.267)$ & 0.272 & $0.060(0.265)$ & 0.820 \\
\hline & Problem solving & $0.097(0.121)$ & 0.424 & $0.290(0.140)$ & 0.039 \\
\hline & Specialized course $(\mathrm{Yes} / \mathrm{No})^{5}$ & $0.084(0.095)$ & 0.381 & $0.084(0.072)$ & 0.245 \\
\hline & Research use in past & $0.221(0.095)$ & 0.020 & $0.248(0.089)$ & 0.006 \\
\hline \multirow[t]{16}{*}{ Unit level covariates $(\mathrm{N}=32)$} & ACT Leadership & $0.233(0.144)$ & 0.106 & $0.437(0.108)$ & $<.0001$ \\
\hline & ACT Culture & $0.834(0.409)$ & 0.042 & $-0.654(0.329)$ & 0.047 \\
\hline & ACT Evaluation & $0.151(0.124)$ & 0.223 & $0.353(0.074)$ & $<.0001$ \\
\hline & ACT Formal Interactions & $-0.053(0.133)$ & 0.691 & $-0.342(0.084)$ & $<.0001$ \\
\hline & ACT Informal Interactions & $0.245(0.137)$ & 0.074 & $0.286(0.085)$ & 0.001 \\
\hline & ACT Social Capital & $-0.076(0.380)$ & 0.841 & $-0.497(0.306)$ & 0.105 \\
\hline & ACT Structural and Electronic Resources & $-0.280(0.146)$ & 0.056 & $0.005(0.104)$ & 0.964 \\
\hline & ACT Organizational Slack-Staff & $-0.060(0.118)$ & 0.612 & $0.027(0.070)$ & 0.702 \\
\hline & ACT Organizational Slack-Space & $-0.074(0.128)$ & 0.562 & $0.247(0.103)$ & 0.016 \\
\hline & ACT Organizational Slack-Time & $0.121(0.302)$ & 0.690 & $-0.113(0.228)$ & 0.620 \\
\hline & Support for innovation & $-0.518(0.273)$ & 0.057 & $-0.244(0.220)$ & 0.268 \\
\hline & Specialty - Critical care ${ }^{6}$ & $0.174(0.172)$ & 0.313 & $0.175(0.146)$ & 0.230 \\
\hline & Medical care & $-0.032(0.113)$ & 0.776 & $0.211(0.093)$ & 0.023 \\
\hline & Average (mean) number of occupied beds & $0.005(0.005)$ & 0.246 & $0.002(0.003)$ & 0.461 \\
\hline & Percentage of baccalaureate nurses & $0.629(0.294)$ & 0.032 & $0.036(0.263)$ & 0.890 \\
\hline & Average patient stay & $-0.026(0.013)$ & 0.050 & $-0.002(0.010)$ & 0.828 \\
\hline \multirow{2}{*}{\multicolumn{2}{|c|}{ QIC \& working correlation }} & \multicolumn{2}{|c|}{$\hat{\rho}=0.00$} & \multicolumn{2}{|c|}{$\hat{\rho}=0.00$} \\
\hline & & \multicolumn{2}{|c|}{$\mathrm{QIC}=723.57$} & \multicolumn{2}{|c|}{$\mathrm{QIC}=704.93$} \\
\hline
\end{tabular}

Bold statistically significant $(\mathrm{p}<.05)$.

${ }^{1}$ Used as continuous to examine the linear trend.

${ }^{2}$ Reference group $=$ Female.

${ }^{3}$ Reference group $=$ Master or higher.

${ }^{4}$ Reference group $=$ Casual.

${ }^{5}$ Reference group $=$ No

${ }^{6}$ Reference group $=$ Surgical care unit. 
measurement of these concepts would be preferable, it is not for the most part possible and if done is prohibitively resource intensive (e.g., informal interactions). Therefore, in order to include unit-level estimates of organizational context dimensions in our models, we needed to obtain data from individual nurses and aggregate these data to the unit-level. Hence, the first step in our analysis was to examine the reliability and validity of the explanatory variables that we planned to aggregate to the unit-level. We calculated four standard empirical aggregation indices for this assessment: intraclass correlation 1, ICC(1) (a measure of individual score variability about the subgroup mean); intraclass correlation 2, ICC (2) (a measure of stability of aggregated data at the group level;); eta-squared, $\eta^{2}$ (the proportion of variance in the individual variable); and omega-squared, $\omega^{2}$ (measures the relative strength of aggregated data as an explanatory variable).

The accepted standards for aggregation are ICC(1) values of 0.10 or greater and ICC(2) values of 0.60 or greater [36,37]. One-way ANOVA with random effects was performed on explanatory variables collected from nurses using 'unit' as the grouping variable. The ANOVA table was then used to calculate the aggregation indices (Table 2). We have reported the calculation and interpretation of these indices previously $[26,38]$.

\section{Modeling approach}

The data collected for this study has a natural hierarchical or clustered structure, meaning nurses were nested within units, which were nested with pediatric hospitals. Each unit constitutes a somewhat unique work context that is shared by the nurses within that unit, and hence nurse responses within a unit may be correlated. There are several approaches to assessing clustered data. In this paper, we used Generalized Estimating Equations (GEE) because this provides the ability to model similarities between nurses that arise from: similarities in measured individual nurse characteristics, similarities resulting from measured unit-level characteristics, and similarities potentially arising from unmeasured unit-level characteristics via what is known as the "working correlation" $[39,40]$. We consider both exchangeable and independent working correlation structures because these comprise the most reasonable alternatives in the context of data clustered for non-longitudinal reasons. The "exchangeable" working correlation estimates the degree of coordination on the dependent variable (over and above the coordination between nurses resulting from the other modeled variables) between all pairs of nurses within a unit, with the same correlation being applied to all pairs of nurses within all units. It estimates the residual degree of similarity between nurses within units where the sources of that similarity remains unknown.
The "independence" GEE model sets the working correlation to zero so there is no residual or beyond-model similarity between nurses within units. This is known as a marginal, or population averaged model [39] and results in consistent and robust estimates despite the unspecified sources of the working correlation. We also report values for Quasi-Akaike's Information Criteria (QIC) regarding model fit. By conducting and examining the findings from both exchangeable and independent working correlation structures, we, in essence, were able to conduct a sensitivity analysis with respect to correlation structure.

\section{Results}

\section{Sample characteristics}

We were able to contact (and thus invite to participate) $80 \%$ of all eligible participants; the number of eligible participants was determined by the study research nurse in consultation with the unit managers while completing the unit profile survey. The overall response rate for nurses (RNs and LPNs) at Year 2 was 39\% $(n=779)$. Missing data was minimal; all nurse cases had $>90 \%$ complete data. Therefore, we did not delete any complete nurse cases based on missing data. Further, in our analyses missing data on any item was treated as missing using listwise deletion. A total of 13 cases were deleted because they did not meet eligibility criteria (i.e., they worked on their nursing unit for less than three months). For the current analysis, we desired a homogenous sample and therefore deleted the 31 LPN cases, leaving us with an analytic sample of 735 RNs. A summary of the demographic data pertaining to the $\mathrm{RN}$ sample is presented in Table 1.

\section{Reliability and validity of aggregated data}

The aggregation statistics supported aggregation to the unit level for the context survey variables theorized to be unit-level (Table 2). These variables had ICC(1) values significantly greater than 0 and several, greater than the 0.10 standard for aggregation indicating a degree of perceptual agreement among the nurses within the units about the values on these explanatory variables. The ICC (2) values were also near or exceeded the accepted standard of 0.60 for unit-level aggregation. Finally, the relative effect sizes $\left(\eta^{2}, \omega^{2}\right)$ were as expected, on average, low to moderate suggesting that as we aggregated these variables, the unit-averages were able to contribute 'low to moderate' portions of the overall variance in the variable.

\section{Results of the GEE analysis}

The GEE analysis for IRU and CRU using an independent or 0.0 working-correlation structure are summarized in Table 3. Initially, we also estimated GEE models employing an exchangeable working correlation but this 
resulted in unreasonable (i.e., negative) working correlation estimates for both IRU and CRU. Therefore, in line with recommendations by Hanley and colleagues [41], we report the results for the independent working correlation models. The estimates from both models (exchangeable and independent working correlations) were highly similar (see Additional file 3).

\section{Instrumental research utilization}

Four variables were identified as significant predictors of IRU at the 5\% level. Significant predictors at the individual-level were: belief suspension-implement (estimate: 0.159) and research use in the past (estimate: 0.221). Significant predictors at the unit (context) level were: culture (estimate: 0.834 ) and proportion of nurses possessing a baccalaureate degree or higher (estimate: 0.629) (Table 3).

\section{Conceptual research utilization}

Ten variables were identified as significant predictors of CRU at the 5\% level. Significant predictors at the individual-level were: belief suspension-implement (estimate: 0.142), problem solving ability (estimate: 0.290), and research use in the past (estimate: 0.248). Significant predictors at the unit (context) level were: leadership (estimate: 0.437), culture (estimate: -0.654), evaluation (estimate: 0.353), formal interactions (estimate: -0.342), informal interactions (estimate: 0.286), organizational slack-space (estimate: 0.247), and unit specialty (medicine compared to surgery) (estimate: 0.211) (Table 3). Overall, only two variables were significant predictors of both IRU and CRU: belief suspension-implement (an individuallevel variable) and culture (a contextual variable).

\section{Discussion}

Our findings raise several important issues for discussion. First, several dimensions of organizational context predict pediatric nurses' use of research findings in clinical practice. Second, there are several differences with respect to which dimensions of context predict IRU compared to CRU. Third, select individual (nurse) characteristics remain significant predictors of nurses' use of research after controlling for organizational context.

\section{The importance of organizational context}

Our findings show that certain dimensions of context predict research use by pediatric nurses. This builds on our previous work in which we reported that pediatric nursing units where nurses reported the highest mean overall research utilization scores (defined as any kind of research use) clustered together on the following contextual factors: unit culture (measured by work creativity, work efficiency, questioning behavior, co-worker support, and the importance nurses place on access to continuing education) and environmental complexity (measured by changing patient acuity and re-sequencing of work) [42]. In a subsequent study, we explored the importance of three dimensions of context (culture, leadership, and evaluation using the measures used in the current study) in relation to instrumental and conceptual research use [26]. In that study, we reported that pediatric nursing units with the highest IRU and CRU scores by nurses had a more positive context (i.e., nurses perceived the culture, leadership, and evaluation of the unit to be positive) compared to units where nurses reported lower research use scores [26]. In our current study, we extended these findings by showing that not only do these dimensions cluster around research use but also that they and additional dimensions of context (i.e., formal interactions, informal interactions, and organizational slack) are important predictors of nurses' instrumental and/or conceptual research use.

In recent years, we have seen a number of studies examining the organizational 'social' context in pediatric social services $[4,5,13,43,44]$. In this work, Glisson and colleagues reported statistically significant associations between organizational climate (defined as 'the way people perceive their work environment' [13]) and child health outcomes (e.g., child psychological functioning) [13]. They also reported that child mental health and social service organizations have a variety of culture profiles and that those profiles are associated with criteria that are important to research implementation $[5,13,44]$. Most recently, Aarons and colleagues [45] conducted a national survey with 1,112 mental health service providers in 100 mental health service (including pediatric) institutions in 26 US states, and found that more proficient organizational cultures and more engaged and less stressful organizational climates were associated with positive clinician attitudes toward adopting evidence-based practice generally. These findings, although conducted in pediatric social services and mental health, align with the findings we report in this paper. Further, our findings also offer the first empirical support of several additional dimensions of context relating to communication (i.e., formal interactions, informal interactions, and organizational slack-space) not previously reported that show promise as important to research use by pediatric healthcare professionals.

\section{Context and CRU}

The contextual dimensions that predicted IRU and CRU differed substantially with only one dimension (culture) displaying significance with both IRU and CRU. The remaining contextual dimensions predicted either IRU or CRU, and substantially more contextual dimensions predicted CRU than IRU. There are several plausible reasons for this finding. First, while not necessary for 
IRU, CRU may be a precursor to IRU in some instances. That is, using research to change one's own thinking, while not necessary may in some cases, lead to using research to change one's behavior. Second, changing the behavior of healthcare professionals (IRU) is both a difficult and multi-component process. It involves consideration of multiple contextual, personal, and behavioral factors. The Theoretical Domains Framework [46,47], for instance identifies 128 constructs tapping different factors important to health professional behavior change. It is therefore plausible that more and different factors than those measured in our study contribute to IRU compared to CRU. Finally, our modeling approach only allowed us to identify which contextual dimensions predict research use and not to determine 'how' context influences research use. It may be that different interactions between contextual dimensions or between context and individual characteristics produce different effects on IRU and CRU or that context indirectly influences IRU through CRU. It is also possible that CRU influences the context variables, or other individual level predictor variables in our models. For example, a nurse who is high in CRU may become higher in 'efficacy', or view the 'leadership' as superior because they are high in CRU.

The majority of our findings revealed that a more positive context predicted higher conceptual research use as hypothesized; however, we did observe two unexpected findings in relation to CRU. Culture (defined as "the way that "we do things in our organizations and work units" with items generally reflecting a supportive work culture [33]) and formal interactions (defined as "formal exchanges that occur between individuals working within an organization (unit) through scheduled activities that can promote the transfer of knowledge" [33]) both displayed negative estimates with CRU indicating a more positive culture and participating in more formal interactions leads to less CRU by nurses. These findings are contrary to theories of context and research use (e.g., PARiHS [34,35]); however, these theories focus on instrumental research use. There is currently no theory and/or empirical research examining contextual predictors of CRU. Therefore, these two findings, while unexpected require replication in future studies before drawing conclusions on these relationships. If the finding persists, it should be investigated qualitatively to better understand the mechanism by which this may occur.

\section{The importance of individual characteristics}

Consistent with a recent systematic review [48], our findings point to the continued importance of individual characteristics. Two individual characteristics in particular, belief suspension and use of research in the past, were significant predictors of both IRU and CRU in our models.

\section{Belief suspension}

The Theory of Planned Behavior (TPB) [49] proposes that motivation determines behavior, and therefore the best predictors of behavior are factors that predict or determine motivation. The theory further asserts that motivation strength is determined by three variables: attitudes, subjective norms, and perceived behavioral control, which in turn are based upon salient beliefs about the behavior [49]. Belief suspension in our study refers to this - an individual's perception of the degree to which they are able to suspend such beliefs in order to use research. Godin and colleagues [50], in a recent systematic review, found healthcare professionals' beliefs about their own capabilities and the consequences of their behavior to be consistently and positively associated, at statistically significant levels, with their motivation to change their behavior. This is in line with our findings that belief suspension is important to nurses' IRU. Future research should focus on determining how belief suspension leads to research use, both instrumentally and conceptually.

\section{Research use in the past}

'Research use in the past' was operationalized as the use of research findings to change practice in the past (i.e. greater than six months ago). Action theories such as Operant Learning Theory (OLT), which are also frequently used to explain behavior, postulate that past behavior is one of the most predictive factors for future behavior. According to OLT, as rewarded behaviours are repeated, they can become 'habitual' in the context in which they are rewarded. As a result, the frequency of past behavior can be a powerful predictor of future behavior [51,52]. Our findings support this with respect to both IRU and CRU. Future research that explores the relationship between past behavior and research use however is needed.

\section{Limitations}

First, our sample is drawn from academically affiliated hospitals only and only includes nurses from pediatric medical, surgical, and intensive care units. Since we were able to contact (to invite to participate) $80 \%$ of all eligible participants, we believe our sample is representative of Canadian pediatric nurses in the medical, surgical, and intensive care units that we surveyed. However, our results should not be generalized beyond these settings or this particular group of nurses. For example, we do not know if the findings would be similar for nurses from community geriatric settings. Second, it is important to note that we did not collect data on several potentially important contextual factors such as overall hospital size, functional differentiation, decision-making structure, data infrastructure, and information systems 
$[17,18]$; future research should incorporate these systemslevel dimensions. Third, as with all modeling techniques, our approach is not without limitation. While the GEE model is an appropriate approach for our data, allowing us to reduce the potential for biased estimates by accounting for the naturally nested structure in our data, it is possible that the proportion of the intra-group variability may change with some explanatory variables, which our GEE models do not allow for. An alternate model such as a mixed effect model with heteroscedasticity would account for such changes in covariance and could be potentially done in the future with larger datasets and fewer explanatory variables (as theory on what dimensions of context predict research use advances, we will be able to narrow the number of explanatory variables to allow such testing).

\section{Conclusions}

Several dimensions of organizational context, as well as individual characteristics, were shown to be important predictors of research use. Interestingly, different dimensions of context predicted IRU and CRU and substantially more dimensions predicted CRU compared to IRU. Unit culture was shown to be a significant predictor of both kinds of research use and formal and informal communications between nurses and other professionals was shown to be important to nurses' CRU. Future research is now needed to understand how these dimensions of context work to influence research use. This study offers empirical evidence of the importance of context to research use by identifying several modifiable dimensions of context that predict IRU and CRU. These findings will be used in our future research to tailor our interventions to the local context with the aim of improving research use by nurses to achieve better patient and eventually, system, outcomes.

\section{Additional files}

Additional file 1: Staff Inclusion and exclusion criteria.

Additional file 2: Description of independent variables.

Additional file 3: GEE Results for independent and exchange working correlation structures.

Competing interests

The authors declare that they have no competing interests.

\section{Authors' contributions}

JES, CAE, SS, GGC and BS participated in designing the study and securing its funding. JES, CAE, LH and SHK designed the analytic plan for the analyses presented in this paper. SHK conducted the statistical analysis; JES, CAE, LH, and SHK interpreted the statistical analysis. JES, SDS and GGC participated in drafting the manuscript. All authors provided critical commentary on the manuscript and approved the final version.

\section{Acknowledgements}

The authors wish to acknowledge additional co-investigators of the CIHR Team in Children's Pain for contributions to this study: Melanie Barwick, Fiona Campbell, Christine Chambers, Janice Cohen, G. Allen Finley, Celeste Johnston, Tricia Kavanagh, Margot Latimer, Shoo Lee, Sylvie Le May, Patrick McGrath, Judith Rashotte, Christina Rosmus, Doris Sawatzky-Dickson, Souraya Sidani, Jennifer Stinson, Robyn Stremler, Anne Synnes, Anna Taddio, Edith Villeneuve, Andrew Willan, Fay Warnock, and Janet Yamada. We also wish to acknowledge Sara Promislow, Project Manager for the CIHR Team in Children's Pain, and Anne-Marie Adachi, Research Manager for Project 2 (described in this manuscript) of the CIHR Team in Children's Pain. The Centre for Computational Biology at The Hospital for Sick Children (Toronto) created, housed, and supported the database. Funding for this project was provided by the Canadian Institutes of Health Research (CIHR) (CTP-79854 and MOP-86605).

\section{Author details}

${ }^{1}$ Clinical Epidemiology Program, Ottawa Hospital Research Institute, Ottawa, Canada. ${ }^{2}$ School of Nursing, Faculty of Health Sciences, University of Ottawa, Ottawa, Canada. ${ }^{3}$ Faculty of Nursing, University of Alberta, Alberta, Canada. ${ }^{4}$ Department of Sociology, Faculty of Arts, University of Alberta, Alberta, Canada. ${ }^{5}$ The Hospital for Sick Children, Toronto, Canada. ${ }^{6}$ Faculty of Nursing, University of Toronto, Toronto, Canada.

Received: 23 January 2013 Accepted: 6 September 2013

Published: 14 September 2013

\section{References}

1. Melnyk BM, Grossman DC, Chou R, Mabry-Hernandez I, Nicholson W, DeWitt TG, Cantu AG, Flores G: Force ftUPST: USPSTF Perspective on EvidenceBased Preventive Recommendations for Children. Pediatrics 2012, 130:e399-e407.

2. Aarons GA: Measuring Provider Attitudes Toward Evidence-Based Practice: Consideration of Organizational Context and Individual Differences. Child Adolesc Psychiatr Clin N Am 2005, 14:255-271.

3. Hemmelgarn AL, Glisson C, James LR: Organizational Culture and Climate: Implications for Services and Interventions Research. Clin Psychol Sci Pract 2006, 13:73-89.

4. Glisson C, Hemmelgarn A: The Effects of Organizational Climate and Interorganizational Coordination on the Quality and Outcomes of Children's Service Systems. Child Abuse Negl 1998, 22:401-421.

5. Glisson C, James LR: The cross-level effects of culture and climate in human service teams. J Org Behav 2002, 23:767-794.

6. Lob SH, Boer JH, Porter PG, Núñez D, Fox P: Promoting Best-Care Practices in Childhood Asthma: Quality Improvement in Community Health Centers. Pediatrics 2011, 128:20-28.

7. Shah S, Sawyer SM, Toelle BG, Mellis CM, Peat JK, Lagleva M, Usherwood TP, Jenkins CR: Improving paediatric asthma outcomes in primary health care: a randomised controlled trial. Med J Aust 2011, 195:405-409.

8. Stout JW, Smith K, Zhou C, Solomon C, Dozor AJ, Garrison MM, MangioneSmith R: Learning from a Distance: Effectiveness of Online Spirometry Training in Improving Asthma Care. Acad Pediatr 2012, 12:88-95.

9. Zhu LM, Stinson J, Palozzi L, Weingarten K, Hogan ME, Duong S, Carbajal R, Campbell FA, Taddio A: Improvements in pain outcomes in a Canadian pediatric teaching hospital following implementation of a multifaceted knowledge translation initiative. Pain Res Manage 2012, 17:173-179.

10. Knowledge to Action: Evidence-Based Health Care in Context. Edited by Dopson S, Fitzgerald L. Oxford: Oxford University Press; 2005.

11. Scott SD, Estabrooks CA, Allen M, Pollock C: A context of uncertainty: how context shapes nurses' research utilization behaviors. Qual Health Res 2008, 18:347-357.

12. Estabrooks CA, Midodzi WK, Cummings GG, Wallin L: Predicting research use in nursing organizations: A multilevel analysis. Nurs Res 2007, 56:S7-S23.

13. Glisson C: Assessing and Changing Organizational Culture and Climate for Effective Services. Res Soc Work Pract 2007, 17:736-747.

14. Johns $\mathrm{G}$ : The essential impact of context on organizational behavior. Acad Manage Rev 2006, 31:386-408.

15. Estabrooks CA: Translating research into practice: Implications for organizations and administrators. Can J Nurs Res 2003, 35:53-68. 
16. Fleuren M, Wiefferink $K$, Paulussen $T$ : Determinants of innovation within health care organizations: Literature review and Delphi study. Int J Qual Health Care 2004, 16:107-123

17. Greenhalgh T, Robert G, Macfarlane F, Bate P, Kyriakidou O: Diffusion of innovations in service organizations: Systematic review and recommendations. Milbank Q 2004, 82:581-629.

18. Kaplan HC, Brady PW, Dritz MC, Hooper DK, Linam WM, Froehle CM, Margolis P: The Influence of Context on Quality Improvement Success in Health Care: A Systematic Review of the Literature. Milbank Q 2010, 88:500-559.

19. Disch J: Clinical Microsystems: The building blocks of patient safety. Creat Nurs 2006, 3:13-14.

20. Godfrey M, Nelson E, Wasson J, Mohr J, Batalden P: Microsystems in healthcare: Part 3. Planning patient-centered services. Jt Comm J Qual Saf 2003, 29:159-170

21. Goldschmidt K, Gordin P: A model of nursing care Microsystems for a large neonatal intensive care unit. Adv Neonatal Care 2006, 6:81-87.

22. Nelson E, Batalden P, Huber T, Mohr J, Godfrey M, Headrick L, Wasson J: Microsystems in health care: Part 1. Learning from high performing front-line clinical units. J Qual Impov 2002, 28:472-493.

23. Nelson E, Godfrey M, Batalden P, Berry S, Bothe A, McKinley K, Melin C, Meuthing S, Moore G, Wasson J, Nolan T: Clinical Microsystems, Part 1. The building blocks of health systems. Jt Comm J Qual Patient Saf 2008, 34:367-378

24. Wasson J, Godfrey M, Nelson E, Mohr J, Batalden P: Microsystems in healthcare: Part 4. Planning patient centered care. Jt Comm J Qual Saf 2003, 29:227-237.

25. Mohr J, Batalden P, Barach P: Integrating patient safety into the clinical microsystem. Qual Saf Health Care 2004, 13:ii34-ii38.

26. Cummings G, Hutchinson A, Scott S, Norton P, Estabrooks C: The relationship between characteristics of context and research utilization in a pediatric setting. BMC Health Serv Res 2010, 10:168.

27. Estabrooks CA: The conceptual structure of research utilization. Nurs Res Health 1999, 22:203-216.

28. Squires J, Estabrooks CA, Newburn-Cook C, Gierl M: Validation of the Conceptual Research Utilization Scale: An Application of the Standards for Educational and Psychological Testing in Healthcare. BMC Health Serv Res 2011, 11:107.

29. Stetler CB: Research utilization: Defining the concept. Image J Nurs Sch 1985, 17:40-44.

30. Stevens BJ, Abbott LK, Yamada J, Harrison D, Stinson J, Taddio A, Barwick M, Latimer M, Scott SD, Rashotte J, Campbell F, Finley GA: Epidemiology and management of painful procedures in children in Canadian hospitals. CMAJ 2011, 183:E403-E410.

31. Estabrooks CA: Modeling the individual determinants of research utilization. West J Nurs Res 1999, 21:758-772

32. Squires J, Hutchinson A, Boström A-M, O'Rourke H, Cobban S, Estabrooks C: To what extent do nurses use research in clinical practice? A systematic review. Implement Sci 2011, 6:21.

33. Estabrooks CA, Squires JE, Cummings GG, Birdsell JM, Norton PG: Development and assessment of the Alberta Context Tool. BMC Health Serv Res 2009, 9:234.

34. Kitson A, Harvey G, McCormack B: Enabling the implementation of evidence based practice: A conceptual framework. Qual Health Care 1998, 7:149-158.

35. Kitson AL, Rycroft-Malone J, Harvey G, McCormack B, Seers K, Titchen A: Evaluating the successful implementation of evidence into practice using the PARiHS framework: Theoretical and practical challenges. Implement Sci 2008, 3.

36. Glick WH: Conceptualizing and measuring organizational and psychological climate: Pitfalls in multilevel research. Acad Manage Rev 1985, 10:601-616

37. James LR: Aggregation bias in estimates of perceptual agreement. J Appl Psychol 1982, 67:219-229.

38. Estabrooks CA, Squires JE, Hutchinson AM, Scott S, Cummings GG, Kang SH, Midodzi WK, Stevens B: Assessment of variation in the Alberta Context Tool: The contribution of unit level contextual factors and specialty in Canadian pediatric acute care settings. BMC Health Serv Res 2011, 11:251.

39. Hardin JW, Hilbe JM: Generalized Estimating Equations. Boca Raton: Chapman and Hall/CRC; 2003.
40. Liang K-Y, Zeger SL: Longitudinal data analysis using generalized linear models. Biometrika 1986, 73:13-22.

41. Hanley JA, Negassa A, Edwardes MD: GEE Analysis of negatively correlated binary responses: a caution. Stat Med 2000, 19:715-722.

42. Estabrooks CA, Scott S, Squires JE, Stevens B, O'Brien-Pallas L, Watt-Watson J, Profetto-Mcgrath J, McGilton K, Golden-Biddle K, Lander J, Donner G, Boschma G, Humphrey CK, Williams J: Patterns of research utilization on patient care units. Implement Sci 2008, 3:31.

43. Glisson C: The organizational context of children's mental health services. Clin Child Fam Psychol Rev 2002, 5:233-253.

44. Glisson C, Green P: The Role of Specialty Mental Health Care in Predicting Child Welfare and Juvenile Justice Out-of-Home Placements. Res SoC Work Pract 2006, 16:480-490.

45. Aarons GA, Glisson C, Green PD, Hoagwood K, Kelleher KJ, Landsverk JA: The organizational social context of mental health services and clinician attitudes toward evidence-based practice: a United States national study. Implement Sci 2012, 7:1.

46. Cane J, O'Connor D, Michie S: Validation of the theoretical domains framework for use in behaviour change and implementation research. Implement Sci 2012, 7:37.

47. Michie S, Johnston M, Abraham C, Lawton R, Parker D, Walker A: Making psychological theory useful for implementing evidence based practice: a consensus approach. Qual Saf Health Care 2005, 14:26-33.

48. Squires J, Estabrooks CA, Gustavsson P, Wallin L: Individual determinants of research utilization by nurses: a systematic review update. Implement SC 2011, 6:1.

49. Ajzen I: The theory of planned behavior. Organ Behav Hum Decis Process 1991, 50:179-211.

50. Godin G, Belanger-Gravel A, Eccles M, Grimshaw J: Healthcare professionals' intentions and behaviours: A systematic review of studies based on social cognitive theories. Implement Sci 2008, 3:36.

51. Ouellette JA, Wood W: Habit and intention in everyday life: The multiple processes by which past behavior predicts future behavior. Psychol Bull 1998, 124:54-74

52. Walker A, Grimshaw J, Johnston M, Pitts N Steen N, Eccles M: PRIME PRocess modelling in ImpleMEntation research: selecting a theoretical basis for interventions to change clinical practice. BMC Health Serv Res 2003, 3:22.

\section{doi:10.1186/1472-6963-13-35}

Cite this article as: Squires et al:: The influence of organizational context on the use of research by nurses in Canadian pediatric hospitals. BMC Health Services Research 2013 13:351.

\section{Submit your next manuscript to BioMed Central and take full advantage of:}

- Convenient online submission

- Thorough peer review

- No space constraints or color figure charges

- Immediate publication on acceptance

- Inclusion in PubMed, CAS, Scopus and Google Scholar

- Research which is freely available for redistribution 